\title{
Prevalencia y riesgos asociados con pacientes adultos con asma de 40 años o más de la Ciudad de México: estudio de base poblacional
}

\author{
Cecilia García-Sancho, D en C,(I) Rosario Fernández-Plata, M en C,(I) David Martínez-Briseño, M en C,(I) \\ Francisco Franco-Marina, M en C, (I) José Rogelio Pérez-Padilla, MC.( ${ }^{(I)}$
}

\section{García-Sancho C, Fernández-Plata R, Martínez-Briseño D, Franco-Marina F, Pérez-Padilla JR. Prevalencia y riesgos asociados con pacientes adultos con asma de 40 años o más de la Ciudad de México: estudio de base poblacional. Salud Publica Mex 20I2;54:425-432.}

\section{Resumen}

Objetivos. Determinar la prevalencia de asma y la asociación entre características sociodemográficas, espirométricas, síntomas respiratorios, calidad de vida y sueño en adultos $\geq 40$ años. Material y métodos. Este informe es parte del estudio PLATINO (Proyecto Latinoamericano de Investigación en Obstrucción Pulmonar), llevado a cabo en la Ciudad de México y área metropolitana en 2003. Se utilizaron modelos de regresión logística ajustados por el diseño del estudio, donde asma fue la variable dependiente y las independientes síntomas respiratorios y características sociodemográficas y clínicas, entre otras. Resultados. La prevalencia de asma diagnosticada por médico fue de $3.3 \%$ en hombres y $6.2 \%$ en mujeres. La función pulmonar disminuyó en asmáticos. En el análisis multivariado, después de ajustar por confusores potenciales, los asmáticos mostraron un mayor riesgo de ronquido más somnolencia excesiva diurna [RM=3.2 (IC95\%|.4-7.4), $p=0.008]$, y mayor frecuencia de inasistencias laborales por problemas respiratorios $[R M=5.1$ (IC95\% 2.5-I0.4), $p<0.000$ I]. Conclusiones. La prevalencia de asma fue de $5 \%$. Los asmáticos mostraron menor calidad de vida y función pulmonar.

Palabras clave: asma; estudios transversales; prevalencia; México
García-Sancho C, Fernández-Plata R, Martínez-Briseño D, Franco-Marina F, Pérez-Padilla JR.

Adult asthma

in Mexico City:

A population-based study.

Salud Publica Mex 20I2;54:425-432.

\section{Abstract}

Objectives. To determine the prevalence of asthma and the association between sociodemographic characteristics, spirometry, respiratory symptoms, quality of life and sleep in adults $\geq 40$ years. Materials and methods. This report is part of our study (Latin American Research Project Obstructive Pulmonary), held in Mexico City and the metropolitan area in 2003. We used logistic regression models adjusted for study design, where asthma was the dependent and independent variable respiratory symptoms, sociodemographic and clinical characteristics among others. Results. The prevalence of physician-diagnosed asthma was: $3.3 \%$ in men and $6.2 \%$ in women. Decreased lung function in asthmatics was observed. In multivariate analysis, after adjusting for potential confounders, asthmatics had a higher risk of excessive daytime sleepiness more snoring [OR $=3.2(95 \% \mathrm{Cl}$ I.4-7.4), $p=0.008]$, and more frequent work absences due to respiratory problems $[\mathrm{OR}=5.1(95 \% \mathrm{Cl} 2.5-10.4), p<0.000 \mathrm{I}]$. Conclusions. The prevalence of asthma was $5 \%$. Asthmatics showed lower quality of life and lung function.

Key words: asthma; cross-sectional studies; prevalence; Mexico

(I) Instituto Nacional de Enfermedades Respiratorias Ismael Cosio Villegas. México.

Fecha de recibido: 3 de octubre de 20II - Fecha de aceptado: 15 de febrero de 2012

Autor de correspondencia: Dra. Ma. Cecilia García Sancho. Instituto Nacional de Enfermedades Respiratorias Ismael Cosio Villegas.

Calzada de Tlalpan 4502. I4080 México, DF.

Correo electrónico: cegarsan@netscape.net 
$E_{s}^{1}$ asma tiene incidencia creciente en todo el mundo. Se considera que existen en el ámbito global 235 millones de personas afectadas. Es también la principal enfermedad crónica en niños. El asma, una afección respiratoria crónica que afecta a 30\% de los niños y $10 \%$ de los adultos del mundo produce una importante carga social y económica, ausentismo escolar y laboral, limitación en las actividades físicas y una mayor utilización de los servicios de salud. ${ }^{1}$

Los principales factores de riesgo asociados con la presentación de asma es la presencia de alergenos en el interior de la vivienda (ácaros del polvo de la casa, en ropa de cama, alfombras y muebles tapizados, y la caspa de los animales, entre otros); alergenos exteriores (pólenes y mohos), y el humo del tabaco e irritantes químicos en el lugar de trabajo. Otros factores desencadenantes pueden incluir el aire frío, la excitación emocional extrema como la ira o el miedo y el ejercicio físico. En algunas personas el asma puede ser desencadenada incluso por ciertos medicamentos como la aspirina y otros fármacos no esteroides antiinflamatorios y betabloqueadores (utilizados para tratar la hipertensión arterial, cardiopatías y la migraña). La urbanización también se ha asociado con un incremento en el asma, sin embargo, la naturaleza exacta de esta relación no está clara. El envejecimiento, la agroindustria (la industria del caucho, en particular), la residencia rural, los antecedentes familiares de asma, los antecedentes de alergias, el aire frío, la inhalación de polvo y gases irritantes, el tabaquismo y la exposición al humo de combustibles de biomasa utilizados para cocinar en muchas partes del mundo se asocian con mayor riesgo de asma. ${ }^{2}$

En México, el asma y el estado asmático constituían la décimotercera causa de morbilidad para 2008. El análisis de la morbilidad por grupo de edad, de acuerdo con los casos notificados a la Secretaría de Salud, muestra que la tasa más alta la tiene el grupo de edad de 0 a 4 años (732.6 casos por 100000 habitantes), seguida por el grupo de 5 a 9 años ( 540.8 casos por 100000 habitantes). Los grupos de edad con menor tasa son el de 15 a 19 años (142.1casos por 100000 habitantes) y el de 20 a 24 años (136.9 casos por 100000 habitantes). A partir de esta edad se incrementa la morbilidad hasta alcanzar la tasa de 215.7 casos por 100000 habitantes en el grupo de edad de 60 a 64 años. En el grupo de 65 y más años, la tasa es de 119.4 casos por 100000 habitantes. En nuestro país, a pesar de que la tasa de mortalidad por asma es baja (<1.5 por 100000 habitantes en 2006), la morbilidad es tan alta que ocasiona una gran carga a los servicios de salud (morbilidad promedio 2003-2009 de 283.3 casos por 100000 habitantes). ${ }^{3}$ Con base en estas cifras se considera que el asma es una causa importante de enfermedad en la población mexicana de adultos y que no ha sido estudiada extensamente.

El conocimiento de la epidemiología del asma es información básica para la planeación de los servicios de salud. En estudios en población adulta realizados en nuestro país, la prevalencia de asma varía de $0.2 \%$ en una población geográficamente definida, ${ }^{4}$ a $8.6 \%$ en una población de 20 a 44 años. ${ }^{5}$ Se desconoce específicamente cual es la prevalencia de la enfermedad a nivel poblacional y en una muestra representativa de la población adulta en nuestro país, es decir, se requiere la realización de encuestas de base poblacional. En este contexto, el objetivo de este estudio fue determinar la prevalencia de asma y la asociación entre el asma y características sociodemográficas, espirométricas, síntomas respiratorios, síntomas relacionados con el sueño, calidad de vida y comorbilidades en adultos $\geq 40$ años, mediante un estudio transversal de base poblacional ubicado en la Ciudad de México y área metropolitana.

\section{Material y métodos}

Este informe es parte del estudio PLATINO (Proyecto Latinoamericano de Investigación en Obstrucción Pulmonar) que es una encuesta transversal de base poblacional que se llevó a cabo en cinco ciudades de Latinoamérica y cuyo principal objetivo fue calcular la prevalencia de enfermedad pulmonar obstructiva crónica (EPOC). Se utilizó la misma estrategia de muestreo multietápica y en conglomerados en las cinco ciudades que participaron originalmente en el estudio. Se trató de un muestreo en tres etapas por conglomerados. En la primera etapa se seleccionaron aleatoriamente 68 geoestadísticas básicas (AGEB) de la zona metropolitana y Ciudad de México. En la segunda etapa, cada AGEB se dividió en cuatro fracciones de las cuales se seleccionó una al azar. De esta sección se levantó un censo de casas habitación. Del listado de casas habitación se seleccionaron 20 al azar para obtener tentativamente 20 sujetos $\geq 40$ años. Se seleccionaron 68 AGEB teniendo en cuenta la estratificación (DF, municipios aledaños) y utilizando una probabilidad de selección proporcional al número de viviendas en cada tracto (tamaño de la AGEB). Se calculó un tamaño de muestra aproximado de 1200 adultos con edad de $\geq 40$ años residentes en las viviendas seleccionadas.

Las áreas metropolitanas se estratificaron primero en ciudad principal y municipios aledaños. Después de la selección de 64 AGEB manteniendo estas proporciones (Ciudad de México, 70\%, municipios aledaños, 30\%), cada AGEB seleccionada se clasificó de acuerdo con el índice de marginación conocido para la zona (alta margi- 
nación 90\%, baja marginación 10\%), tratando de guardar la proporción del índice de marginación estimado para la zona. Tomando como supuesto que la proporción de personas con edad $\geq 40$ años es de $20 \%$ en un hogar de cinco personas, se asumió que en cada una de las 20 casas habitación seleccionada al azar residía un sujeto con edad $\geq 40$ años. De esta forma se contactaron 1066 viviendas en las que residían 1452 personas de 40 años o más, siendo posible estudiar a 1063 de ellas (tasa de respuesta de 73.2\%). La metodología del estudio PLATINO se ha descrito anteriormente. ${ }^{6}$ Aquí se presentan los resultados para 1063 personas residentes en la Ciudad de México y área metropolitana que participaron en el estudio PLATINO.

Todos los participantes firmaron la carta de consentimiento informado. A los que aceptaron participar se les aplicó el cuestionario PLATINO y se les hizo una evaluación antropométrica para calcular el índice de masa corporal (IMC): peso, talla y una espirometría, la cual se realizó de acuerdo con las recomendaciones internacionales de la Sociedad Torácica Americana (ATS, por sus siglas en inglés). ${ }^{7}$ La EPOC fue definida por el criterio de la Gold Iniciative for Chronic Obstructive Lung Disease (GOLD, por sus siglas en inglés): un cociente VEF1/CVF $<0.70$ (VEF1: volumen espiratorio forzado en el primer segundo y CVF: capacidad vital forzada). En este análisis se consideró que los individuos con un cociente VEF1/ $\mathrm{CVF}<0.70$ prebroncodilatador (pre-BD) pero con un cociente VEF1/FVC $\geq 0.70$ postbroncodilatador (postBD) tenían obstrucción reversible. La respuesta aguda al broncodilatador (salbutamol $200 \mu \mathrm{g}$ ) fue evaluada con base en tres definiciones: a) un incremento $\geq 12 \%$ en el VEF1 o CVF; b) un incremento en el VEF1 $\geq 15 \%$ en relación con el valor basal y c) un incremento $\geq 10 \%$ del valor predicho. ${ }^{8}$ Las entrevistas y exámenes se hicieron por personal debidamente entrenado.

El cuestionario PLATINO incluye secciones de varios cuestionarios internacionales que evalúan salud respiratoria. ${ }^{9-11}$ Para evaluar el estado de salud general de una población se ha usado el cuestionario SF-36 $6^{12}$ (por sus siglas en inglés short-form), sin embargo, en este estudio se utilizó la versión reducida que es el SF-12, ${ }^{13}$ que se aplica en menor tiempo y que ha sido recomendado para una muestra de 500 personas o más.

La información sobre el diagnóstico médico de asma se basó en la pregunta: “¿Algún médico le ha dicho que tenía o tiene usted asma?" Para aquellos que respondieron que sí, los encuestadores preguntaron acerca del uso y tipo de medicamentos que el individuo utilizaba para tratar la enfermedad. Además de la presencia de síntomas respiratorios, el cuestionario incluyó preguntas de las características sociodemográficas, exposiciones dentro y fuera de la vivienda, exposiciones ocupacionales a humo y gases, síntomas autorreportados relacionados con el sueño, e inasistencias laborales debidas a problemas respiratorios. Se interrogó asimismo la presencia de comorbilidades (diagnóstico médico de problemas cardíacos, hipertensión, diabetes, gastritis y úlcera, y accidente cerebrovascular). Dos especialistas en medicina del sueño hicieron una recopilación de preguntas previamente aplicadas en encuestas internacionales y que fueron incorporadas al cuestionario PLATINO. ${ }^{14,15}$ El protocolo del estudio y la carta de consentimiento informado fueron aprobados por el Comité de Ciencia y Bioética en Investigación del INER.

\section{Análisis estadístico del estudio}

La prevalencia de asma se calculó con base en la población que refirió tener un diagnóstico previo de asma hecho por un médico. Los síntomas autorreportados asociados con el sueño se clasificaron de acuerdo con lo que han reportado los investigadores del grupo PLATINO. ${ }^{10}$ Se hizo un análisis bivariado para identificar las características asociadas con los pacientes con asma. Después del análisis bivariado se realizaron modelos de regresión logística múltiple ajustados por el diseño del estudio con el método de inclusión. La variable dependiente fue asma y se controló por edad, sexo, índice de masa corporal, y exposiciones ocupacionales y ambientales. Además se evaluaron los efectos de interacción y confusión (STATA, versión 9.0).

\section{Resultados}

La población elegible para el estudio en la Ciudad de México fue de 1452 personas de ambos sexos con edad $\geq 40$ años, de las que fue posible estudiar a 1063 (tasa de respuesta de 73.2\%). De los 1063 participantes, no se observaron diferencias en la tasa de respuesta por sexo o por estado asmático. No contamos con información de las 389 personas que no participaron en el estudio. La media de edad para el grupo fue de $55.9( \pm \mathrm{DE} 11.9)$ años y la proporción de hombres fue de $42.3 \%$. La media de escolaridad formal fue de 7.1 ( \pm DE 5.0) años y el IMC de 28.8 ( \pm DE 5.1). La prevalencia de tabaquismo en nuestra población fue de $19 \%$.

La prevalencia de asma diagnosticada por un médico fue de $5 \%$; de $3.3 \%$ en hombres y $6.2 \%$ en mujeres $(p=0.03)$. En el análisis bivariado, los pacientes con asma refirieron una mayor frecuencia de síntomas respiratorios, uso de oxígeno en casa, inasistencias al trabajo por problemas respiratorios, diabetes mellitus y gastritis o úlcera diagnosticadas por el médico (cuadro I).

El promedio de cigarrillos fumados por día en los pacientes con asma fue mayor que en el resto de la 


\section{CuAdro I \\ Características sociodemográficas y clínicas de la población de adUltos $\geq \mathbf{4 0}$ años con y Sin asma de la Ciudad de México. 2003}

\begin{tabular}{|c|c|c|c|c|}
\hline Característica & $\begin{array}{l}\text { Casos de asma* } \\
\quad(n=53)\end{array}$ & $\begin{array}{l}\text { Población sin asma* } \\
\qquad(n=1010)\end{array}$ & $R M(I C 95 \%)^{\ddagger}$ & $p$ \\
\hline Edad & $57.0( \pm 10.2)$ & $55.9( \pm 12.0)$ & $1.007(0.98-1.02)$ & 0.4 \\
\hline Sexo masculino & $15 / 53(28.3)$ & $435 / 1010(43.1)$ & $0.5(0.3-0.9)$ & 0.03 \\
\hline Años de educación formal & $6.8( \pm 5.8)$ & $7.1(4.9)$ & $0.99(0.92-1.06)$ & 0.8 \\
\hline \multicolumn{5}{|l|}{ Síntomas últimos 12 meses } \\
\hline Tos sin estar resfriado & $20 / 53(37.7)$ & $128 / 1010(12.7)$ & $4.2(2.3-7.6)$ & $<0.0001$ \\
\hline Catarro sin resfrío & $17 / 53(32.1)$ & $147 / 1010(14.6)$ & $2.8(I .6-4.7)$ & $<0.0001$ \\
\hline Silbido & $25 / 53(47.2)$ & $157 / 1010(15.5)$ & $4.9(2.6-8.9)$ & $<0.0001$ \\
\hline Silbido sólo estando resfriado & $11 / 25(44.0)$ & $100 / 157(63.7)$ & $0.4(0.2-1.1)$ & 0.08 \\
\hline Silbido con disnea & $7 / 25(28.0)$ & $39 / 155(25.1)$ & $\mathrm{I} .2(0.5-2.7)$ & 0.7 \\
\hline Tos o flema o silbido o disnea & $49 / 53(92.5)$ & $627 / 1010(62.1)$ & $7.5(2.7-20.7)$ & $<0.0001$ \\
\hline Disnea al caminar una subida pequeña & $39 / 53(73.6)$ & $516 / 1005(51.3)$ & $2.6(I .5-4.5)$ & 0.001 \\
\hline Camina más lento que personas de su misma edad & $\mathrm{I} / \mathrm{I} 4(7 . \mathrm{I})$ & $1 \mathrm{I} / 487(2.3)$ & $3.3(0.4-30.9)$ & 0.3 \\
\hline Se detiene al caminar 100 metros & $\mathrm{I} / \mathrm{I} 3(7.7)$ & $1 \mathrm{I} / 472(2.3)$ & $3.5(0.4-32)$ & 0.3 \\
\hline Espirometría previa & 9/53(I7.0) & $64 / 1010(6.3)$ & $3.0(1.3-7.0)$ & 0.01 \\
\hline Uso de oxígeno en casa & $2 / 53(3.8)$ & $8 / 1008(0.8)$ & $4.9(1.02-23.4)$ & 0.04 \\
\hline Inasistencias laborales debidas a problemas respiratorios & $17 / 53(32.1)$ & $52 / 1010(5.2)$ & $8.7(4.6-16.5)$ & $<0.0001$ \\
\hline Hipertensión arterial diagnosticada por médico & $22 / 53(41.5)$ & $310 / 1010(30.7)$ & $1.6(0.9-2.9)$ & 0.1 \\
\hline Diabetes mellitus diagnosticada por médico & $14 / 53(26.4)$ & $148 / 1010(14.7)$ & $2.1(1.2-3.6)$ & 0.009 \\
\hline Gastritis o úlcera diagnosticada por médico & $25 / 53(47.2)$ & $332 / 1010(32.9)$ & I.8(I.04-3.2) & 0.03 \\
\hline Con obesidad, diabetes mellitus, cardiopatía e hipertensión arterial & $2 / 53(3.8)$ & $14 / 1010(1.4)$ & $2.8(0.6-13.2)$ & 0.2 \\
\hline Índice de masa corporal & $28.4( \pm 5.3)$ & $28.8( \pm 5.0)$ & $0.98(0.92-1.4)$ & 0.7 \\
\hline
\end{tabular}

* Los datos corresponden a n(\%) o a media ( \pm desviación estándar)

¥ Razones de momios crudas ajustadas por diseño del estudio.

población $16.6( \pm \mathrm{DE} 17.2)$ versus $5.6( \pm \mathrm{DE} 5.9), p=0.003$. Los pacientes con asma tuvieron con mayor frecuencia una percepción de mala salud, depresión, angustia, falta de energía, o una combinación de estas tres últimas. En el mismo análisis bivariado, los pacientes con asma mostraron mayor frecuencia de ronquido, cansancio, somnolencia diurna excesiva y de la combinación de ronquido habitual más somnolencia diurna excesiva. El tiempo promedio de sueño fue de 6.2 horas $( \pm \mathrm{DE}$ 1.6) en los pacientes con asma versus $7.0( \pm \mathrm{DE} 1.5)$ horas en el resto de la población, $p<0.0001$ (cuadro II).

De 964 personas con espirometría evaluable (90.7\%), $33 \%$ resultó normal; $5.2 \%$ mostró obstrucción reversible y 7.8\% obstrucción irreversible (datos no mostrados). La comparación entre los sujetos con y sin asma mostró que los primeros tenían una función pulmonar reducida: cociente VEF1/CVF 74.6 ( \pm 9.6$)$ versus 77.9 ( \pm 7.1 .), $p=0.005$ (cuadro III).
En el análisis multivariado, después de controlar por edad, sexo, IMC, diabetes tipo 2, tabaquismo (paquetes / año) y cansancio, los pacientes con asma mostraron un riesgo tres veces mayor de presentar ronquido más somnolencia diurna excesiva $(22.6 \%$ versus $7.7 \%$, $\mathrm{RM}=3.2$ (IC95\% 1.4-7.4) $p=0.008$ ); y cinco veces mayor de inasistencias laborales por problemas respiratorios (32.1\% versus $5.2 \%$; $R M=5.1$ (IC95\% 2.5-10.4), $p<0.0001$ ) (cuadro IV).

\section{Discusión}

La prevalencia de asma en población adulta residente en la Ciudad de México y área metropolitana es de 5\%, significativamente menor en hombres $(3.3 \%)$ que en mujeres $(6.2 \%), p=0.03$. En nuestro estudio la obesidad (IMC $\geq 30)$ se asoció con asma en mujeres pero no en hombres. Los síntomas autorreportados relacionados 


\section{Cuadro II \\ Características de Calidad de vida, sueño y exposiciones de la población de adultos $\geq 40$ años con y Sin asma DE LA CIUDAD DE MÉXICO. 2003}

\begin{tabular}{|c|c|c|c|c|}
\hline Característica & $\begin{array}{l}\text { Casos de asma* } \\
\quad(n=53)\end{array}$ & $\begin{array}{l}\text { Población sin asma* } \\
\qquad(n=1010)\end{array}$ & RM (IC95\%) $)^{\ddagger}$ & $p$ \\
\hline \multicolumn{5}{|l|}{ Exposiciones } \\
\hline Exposición ocupacional a polvo mayor a 5 años & $13 / 53(24.5)$ & $302 / 1010(29.9)$ & $0.8(0.4-1.5)$ & 0.4 \\
\hline Fumó alguna vez & $25 / 53(47.2)$ & $447 / 1063(44.3)$ & I.I (0.6-I.9) & 0.7 \\
\hline Fuma actualmente & $12 / 53(22.6)$ & $258 / 1010(25.5)$ & $0.9(0.4-1.7)$ & 0.6 \\
\hline Exfumador & $13 / 53(24.5)$ & $189 / 1010(18.7)$ & I.4(0.8-2.5) & 0.2 \\
\hline Edad de inicio & $20.6( \pm 6.3)$ & $20.3( \pm 8.5)$ & $1.003(0.9-1.06)$ & 0.9 \\
\hline Cuantos cigarrillos en promedio al día & $16.6( \pm 17.2)$ & $5.6( \pm 5.9)$ & $1.10(1.03-1.2)$ & 0.003 \\
\hline \multicolumn{5}{|l|}{ Tabaquismo } \\
\hline Paquetes año & $3.8( \pm 10.6)$ & $2.5( \pm 9.8)$ & $1.01(0.99-1.02)$ & 0.2 \\
\hline \multicolumn{5}{|l|}{ Calidad de vida } \\
\hline Percepción de mala salud & $39 / 53(73.6)$ & $588 / 1010(58.2)$ & $2.0(1.2-3.4)$ & 0.01 \\
\hline Con depresión & $13 / 53(24.5)$ & $104 / 1010(10.3)$ & $2.8(1.4-5.9)$ & 0.007 \\
\hline Con angustia & $1 \mathrm{I} / 53(28.8)$ & $122 / 1010(12.1)$ & $1.9(1.005-3.6)$ & 0.04 \\
\hline Sin energía & $14 / 53(26.4)$ & $140 / 1010(13.9)$ & $2.2(1.2-4.3)$ & 0.01 \\
\hline Angustiado, deprimido, sin energía & $5 / 53(9.4)$ & $26 / 1010(2.6)$ & $3.9(1.5-10.6)$ & 0.007 \\
\hline \multicolumn{5}{|l|}{ Síntomas relacionados al sueño } \\
\hline Toma siesta & 9/53(I7.0) & $217 / 1007(21.6)$ & $0.7(0.3-1.7)$ & 0.5 \\
\hline Con insomnio & $23 / 53(43.4)$ & $350 / 1010(34.7)$ & I.4(0.8-2.5) & 0.2 \\
\hline Ronquido & $37 / 53(69.8)$ & $546 / 1010(54.1)$ & $2.0(0.04-3.7)$ & 0.03 \\
\hline Ahogo & $16 / 53(30.2)$ & $214 / 1010(21.2)$ & I.6(0.9-2.9) & 0.1 \\
\hline Cansado & $26 / 53(49.1)$ & $327 / 1010(32.4)$ & $2.0(1.2-3.5)$ & 0.01 \\
\hline Apnea & $3 / 53(5.7)$ & $99 / 1010(9.8)$ & $0.6(0.2-1.9)$ & 0.3 \\
\hline Somnolencia excesiva diurna & $17 / 53(32.1)$ & $172 / 1009(17.1)$ & $2.3(1.2-4.3)$ & 0.01 \\
\hline Ronquido habitual, somnolencia excesiva diurna & $12 / 53(22.6)$ & $78 / 1009(7.7)$ & $3.5(1.6-7.4)$ & 0.001 \\
\hline Horas de sueño promedio & $6.2( \pm 1.6)$ & $7.0( \pm 1.5)$ & $0.7(0.6-0.9)$ & $<0.0001$ \\
\hline
\end{tabular}

* Los datos corresponden a $\mathrm{n}(\%)$ o a media ( \pm desviación estándar)

‡ Razones de momios crudas ajustadas por diseño del estudio.

con el sueño fueron ocho veces más frecuentes en los pacientes con asma que en la población general.

Este es el primer estudio de base poblacional en México que determina la prevalencia de asma en adultos, la cual fue de 5\%. En estudios en población adulta realizados en nuestro país, la prevalencia de asma varía de $0.2 \%$ en una población geográficamente definida, ${ }^{14}$ a $8.6 \%$ en población de 20 a 44 años, ${ }^{15}$ sin embargo, estos estudios no son comparables al nuestro por las diferencias en su diseño epidemiológico y en la edad de las poblaciones estudiadas.

Desde el estudio del International Study of Asthma and Allergies in Childhood (ISAAC, por sus siglas en inglés) es sabido que la prevalencia de asma y alergias varía considerablemente en el mundo de 0.8 a $32.6 \%$ por motivos conocidos de manera incompleta. ${ }^{16} \mathrm{En}$ general la prevalencia es menor en áreas rurales que en urbanas, y en México se ha documentado que es menor en el altiplano que a nivel del mar. ${ }^{17}$ La variabilidad se incrementa mucho más cuando se utilizan criterios heterogéneos de diagnóstico.

En nuestro estudio encontramos una mayor frecuencia de asma en mujeres, lo que confirma los resultados de otros investigadores. En Australia, en población adulta se encontró una tendencia al incremento en las tasas anuales de asma significativamente mayor en mujeres, y este incremento se produjo simultáneamente con el aumento en la prevalencia de obesidad y diabetes 


\section{Cuadro III \\ Características espirométricas de la población de adULtos $\geq \mathbf{4 0}$ años CON Y Sin ASMA de la Ciudad de México y del uso de Servicios de salud. 2003}

\begin{tabular}{|c|c|c|c|c|}
\hline Característica & $\begin{array}{c}\text { Casos de asma* } \\
\quad(n=50)\end{array}$ & $\begin{array}{c}\text { Población sin asma* } \\
\qquad(n=950)\end{array}$ & RM $(I C 95 \%)^{\S}$ & $p$ \\
\hline \multirow[t]{2}{*}{ VEFI pre-broncodilatador \% $\mathrm{P}^{\ddagger}$} & $90.2( \pm 24.0)$ & $97.6( \pm 16.0)$ & & \\
\hline & $(17.7-132.2)$ & $(24.1-160.9)$ & $0.97(0.95-0.99)$ & 0.02 \\
\hline \multirow[t]{2}{*}{ CVF pre-broncodilatador \% $\mathrm{P}^{\ddagger}$} & $94.2( \pm 22.3)$ & $98.0( \pm 15.0)$ & & \\
\hline & $(29.8-135.6)$ & $(3||-.\mid 55.3)$ & $0.98(0.95-1.01)$ & 0.3 \\
\hline \multirow[t]{2}{*}{$\mathrm{VEFI/CVF}$} & $74.6( \pm 9.6)$ & $77.9( \pm 7.1)$ & & \\
\hline & $(40.9-87.9)$ & $(33.9-99.9)$ & $0.95(0.92-0.98)$ & 0.005 \\
\hline Obstrucción pre-broncodilatador & $12 / 50(24.0)$ & $105 / 950(I I .1)$ & $2.5(1.3-4.9)$ & 0.007 \\
\hline Respuesta a broncodilatador en VEFI o CVF & $7 / 45(\mid 5.6)$ & $69 / 919(7.5)$ & $2.3(0.91-5.6)$ & 0.07 \\
\hline \multicolumn{5}{|l|}{ Cociente VEFI/CVF <límite inferior normal } \\
\hline Pre-broncodilatador & $6 / 45(12.0)$ & $45 / 950(4.7)$ & $2.7(0.9-7.7)$ & 0.05 \\
\hline Cociente VEFI/CVF <LIN post-broncodilatador & $4 / 45(8.9)$ & $29 / 919(3.2)$ & $3.0(0.9-10.5)$ & 0.08 \\
\hline \multicolumn{5}{|l|}{ Servicios de salud } \\
\hline Tiene seguro de salud & $34 / 53(64.2)$ & $607 / 1010(60.1)$ & $1.2(0.7-2.1)$ & 0.5 \\
\hline Número de veces que fue al médico en los últimos 12 meses & $2.3( \pm 1.6)$ & $2.0( \pm 1.7)$ & $\mathrm{I} . \mathrm{I}(0.7-\mathrm{I} .8)$ & 0.7 \\
\hline Se hospitalizó durante los últimos 12 meses por causas respiratorias & $5 / 53(9.4)$ & $2 / 1010(0.2)$ & $52.5(9.8-280)$ & $<0.0001$ \\
\hline Uso previo de broncodilatadores inhalados & $3 / 53(5.7)$ & $8 / 100 I(0.8)$ & $7.5(2.2-25.5)$ & 0.002 \\
\hline Uso de esteroides inhalados y orales & $1 / 53(1.9)$ & $2 / 1010(0.2)$ & $9.7(0.8-116.8)$ & 0.07 \\
\hline Uso de cualquier medicamento para problemas respiratorios & $3 / 53(5.7)$ & $62 / 1010(6.1)$ & $0.9(0.3-3.1)$ & 0.9 \\
\hline
\end{tabular}

* Los datos corresponden a n(\%) o a media ( \pm desviación estándar)

¥ Porcentaje del predicho

$\S$ Razones de momios crudas ajustadas por diseño del estudio.

y con la menor realización de ejercicio vigoroso. ${ }^{18}$ Esto se ha atribuido a las diferencias en la distribución de grasa corporal entre hombres y mujeres.

La asociación entre obesidad y asma en adultos ha sido reportada previamente. ${ }^{19} \mathrm{Al}$ igual que en otros estudios, encontramos que la obesidad se asoció con asma sólo en las mujeres. ${ }^{20}$ Se ha encontrado, además, que la obesidad en mujeres asmáticas se asocia con obstrucción pulmonar, pero no con inflamación de las vías aéreas. ${ }^{21}$ También se ha descrito que la asociación entre obesidad y adipocinas es más fuerte en mujeres que en hombres con asma, debido probablemente a las diferencias por sexo en la distribución de la grasa ectópica. ${ }^{22}$ En un estudio clínico realizado en pacientes con asma, la masa magra total estuvo más fuertemente asociada con asma que la masa de grasa total en mujeres, lo que sugiere que la grasa ectópica dentro de los tejidos magros es responsable de la asociación obesidad-asma en mujeres. ${ }^{23}$ Esta asociación es importante ya que se ha descrito un incremento reciente de asma y obesidad en la población mexicana, con el subsecuente incremento en el costo de la atención médica y hospitalaria.
La presencia de enfermedad pulmonar obstructiva crónica (EPOC) ( $p=0.3)$; enfermedad cardiaca $(p=0.5)$; hipertensión arterial $(p=0.09)$; cáncer pulmonar $(p=0.7)$; accidente vascular cerebral $(p=0.9)$ y tuberculosis $(p=0.5)$ no fueron más frecuentes entre los pacientes con asma que en la población general. Los pacientes con asma presentaron mayor frecuencia de diabetes tipo 2 (26.4 vs. $14.7 \%, p=0.02)$, y gastritis o úlcera (47.2 vs. $32.9 \%$, $p=0.03$ ), enfermedades que se encuentran con mayor frecuencia en los pacientes con asma de acuerdo con la literatura internacional. El que no existan diferencias significativas en la frecuencia de enfermedades crónicas revela que los casos y la población general reportaron de manera similar dichas enfermedades, es decir, sin que se observara un mayor reporte entre los pacientes con asma. Esta asociación entre diabetes tipo 2, úlcera gástrica y asma ya había sido reportada anteriormente por otros investigadores. ${ }^{24-27}$

En nuestro estudio también encontramos que los síntomas autorreportados relacionados con el sueño fueron tres veces más frecuentes en los pacientes con asma que en la población general. Estos datos confirman 


\section{Cuadro IV

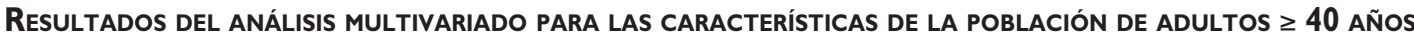 CON Y SIN ASMA DE LA CIUdAd de MéXICO. 2003}

\begin{tabular}{|c|c|c|c|c|}
\hline Característica & $\begin{array}{c}\text { Casos con asma } \\
\quad(n=53)\end{array}$ & $\begin{array}{l}\text { Población sin asma } \\
\qquad(n=1010)\end{array}$ & $\begin{array}{l}\text { RM cruda } \\
\text { (IC95\%) }\end{array}$ & $\begin{array}{l}\text { RM ajustada } \\
\text { (IC95\%) }\end{array}$ \\
\hline \multirow[t]{2}{*}{ Edad } & $57.0( \pm 10.2)$ & $55.9( \pm 12.0)$ & $\mathrm{I} .007(0.98-1.02)$ & $\mathrm{I} .005(0.9-1 . \mathrm{I})$ \\
\hline & & & $p=0.4$ & $p=0.9$ \\
\hline \multirow[t]{2}{*}{ Sexo } & $15 / 53(28.3)$ & $435 / 1010(43.1)$ & $0.5(0.3-0.9)$ & $0.5(0.1-1.9)$ \\
\hline & & & $p=0.04$ & $p=0.3$ \\
\hline \multirow[t]{2}{*}{ Îndice de masa corporal } & $6.8( \pm 5.8)$ & $7.1(4.9)$ & $1.0(0.9-1.1)$ & $0.9(0.7-1.04)$ \\
\hline & & & $p=0.7$ & $p=0.1$ \\
\hline \multirow[t]{2}{*}{ Silbido } & $25 / 53(47.2)$ & $157 / 1010(15.5)$ & $4.9(2.6-8.9)$ & $12.7(\mid .9-83.1)$ \\
\hline & & & $p<0.0001$ & $p=0.009$ \\
\hline \multirow[t]{2}{*}{ Diabetes mellitus diagnosticada por médico } & $14 / 53(26.4)$ & $148 / 1010(14.7)$ & $2.1(1.2-3.6)$ & I.3(0.3-6.3) \\
\hline & & & $p=0.009$ & $p=0.8$ \\
\hline \multirow[t]{2}{*}{ Inasistencias laborales debidas a problemas respiratorios } & $17 / 53(32.1)$ & $52 / 1010(5.2)$ & $8.7(4.6-16.5)$ & $5.1(2.5-10.4)$ \\
\hline & & & $p<0.0001$ & $p<0.0001$ \\
\hline Tabaquismo & $3.8( \pm 10.6)$ & $2.5( \pm 9.8)$ & $1.01(0.99-1.02)$ & $0.7(0.5-1.2)$ \\
\hline Paquetes año & & & $p=0.2$ & $p=0.2$ \\
\hline \multirow[t]{2}{*}{ Somnolencia excesiva diurna } & $17 / 53(32.1)$ & $172 / 1009(\mid 7.1)$ & $2.3(1.2-4.3)$ & $11.9(1.001-140)$ \\
\hline & & & $p=0.01$ & $p=0.05$ \\
\hline \multirow[t]{2}{*}{ Ronquido habitual, somnolencia excesiva diurna } & $12 / 53(22.6)$ & $78 / 1009(7.7)$ & $3.5(1.6-7.4)$ & $3.2(1.4-7.4)$ \\
\hline & & & $p=0.00 \mathrm{I}$ & $p=0.008$ \\
\hline \multirow[t]{2}{*}{ Sin energía } & $14 / 53(26.4)$ & $140 / 1010(13.9)$ & $2.2(1.2-4.3)$ & $8.6(1.8-40.1)$ \\
\hline & & & $p=0.01$ & $p=0.007$ \\
\hline \multirow[t]{2}{*}{ Cansancio } & $26 / 53(49.1)$ & $327 / 1010(32.4)$ & $2.0(1.2-3.5)$ & $0.6(0.1-2.1)$ \\
\hline & & & $p=0.01$ & $p=0.4$ \\
\hline
\end{tabular}

* Razones de momios crudas y ajustadas, ajustadas por diseño del estudio.

lo descrito por otros investigadores que observaron que la disminución de los niveles de control del asma se asocia con una mayor prevalencia de problemas del sueño, depresión, discapacidad funcional, efecto sobre el trabajo y las actividades habituales. ${ }^{29}$ Esta asociación también podría estar mediada por la obesidad.

En nuestro estudio, el grupo de pacientes con asma mostró una mayor frecuencia de tos, silbido y de la combinación de tos o flema o silbido o disnea en comparación con el grupo de individuos sin asma. A pesar de que la población con asma fumó un mayor promedio de cigarrillos por día, no se observaron diferencias entre las proporciones de fumadores, exfumadores y fumadores actuales cuando se compararon con el resto de la población. En relación a los medicamentos, únicamente el uso de broncodilatadores inhalados fue significativamente mayor entre los sujetos con asma.

No obstante la fortaleza del diseño de encuesta transversal de base poblacional, este estudio tiene la limitación principal de que la evaluación del asma se hizo mediante cuestionario. A pesar de que esta pregunta ha sido validada por estudios de base poblacional en todo el mundo, es conveniente incorporar criterios adicionales que permitan un análisis más detallado como la hiperreactividad bronquial, pruebas de alergia, exposición a contaminantes intra y extramuros, dieta y factores hereditarios. Si bien estos estudios añaden información valiosa a una simple encuesta tienen el inconveniente de ser estudios con mayor potencial de eventos adversos que no serán aprobados fácilmente por un comité de ética ni por la población de estudio.

En este estudio de base poblacional encontramos que la prevalencia de asma diagnosticada por un médico fue de $5 \%$ en población adulta y que el asma es una condición médica que impacta significativamente la calidad de vida, el estado de salud general y el sueño. Los resultados de esta investigación apoyan la adopción de un enfoque integrado en la identificación y control del asma en esta población.

Declaración de conflicto de intereses. Los autores declararon no tener conflicto de intereses. 


\section{Referencias}

I. World Health Organization. Asthma, fact sheet no. 307, May 201 I. [Actualizado el I de mayo de 20I I]. Disponible en: http://www.who.int/ mediacentre/factsheets/ts307/en/index.html 2. Ding YP, Yao HX, Tang XL, He HW, Shi HF, Lin L, et al. An epidemiology study of bronchial asthma in the Li ethnic group in China. Asian Pac J Trop Med 2012;5(2):|57-|6|.

3. Secretaría de Salud. Sistema Nacional de Vigilancia Epidemiológica. Epidemiología. Anuario de morbilidad 2009. [Actualizado el 10 de agosto de $20 \mathrm{II}$, consultado el 26 de abril de $20 \mathrm{II}$. Disponible en: http://www. dgepi.salud.gob.mx/2010/plantilla/inicio_anuarios.html,

4. Vázquez Nava F, Saldívar González AH, Córdova Fernández A, Vázquez Rodríguez EM, García Maldonado G, Martínez Perales GM, et al. Association among familial atopy, smoking (passive and active), allergic rhinitis, labor environment and adult asthma. Rev Alerg Mex 2008;55:222-228. 5. Nadal-Blanco MJ, Magro-Perteguer R, Villena-Romero R, Gil-Zarzosa L, Sánchez-Hernández I, Guinea-Jaime J. Prevalence of symptoms related to asthma in Guadalajara. Arch Bronconeumol 1998;34:289-294.

6. Menezes AM, Victora CG, Perez-Padilla R, Platino Team. The Platino project: methodology of a multicenter prevalence survey of chronic obstructive pulmonary disease in major Latin American cities. BMC Med Res Methodol 2004;4: I5. doi:I0.I I86/I47I-2288-4-5, [consultado el 26 de abril de 201 I]. Disponible en: http://www.biomedcentral.com/I47I-2288/4/I5 7. Miller MR, Hankinson J, Brusasco V, Burgos F, Casaburi R, Coates A, et al. Standardisation of spirometry. Eur Respir J 2005;26:319-338. 8. Montes de Oca M, Pérez Padilla R, Tálamo C, Halbert RJ, Moreno D, López MV, et al. For the Platino Team. Acute bronchodilator responsiveness in subjects with and without airflow obstruction in five Latin American cities: The Platino study. Pulm Pharmacol Ther 2010;23:29-35. 9. Ferris BG. Epidemiology Standardization Project (American Thoracic Society) Am Rev Respir Dis 1978; I 18:1-120.

10. The European Community Respiratory Health Survey II. Eur Respir J 2002; 20:107|-1079.

II. BC Cancer Research Centre. Lung Health Study Questionnaire. Vancouver: BC Cancer Research Centre, 2004.

12. Ware JE, Sherbourne CD. The MOS 36-item short-form health survey (SF-36). I. Conceptual framework and item selection. Med Care. 1992:30:473-483.

13. Ware JE, Kosinski M, Keller SD. How to score the SFI2 Physical and Mental Health Summary Scales. 2nd edition. Boston: The Health Institute, New England Medical Center, 1995

14. Bouscoulet LT, Vázquez-García JC, Muiño A, Márquez M, López MV, de Oca MM, et al. Prevalence of sleep related symptoms in four Latin American cities. Clin Sleep Med 2008;4:579-585.
15. México. Proyecto Latinoamericano de Investigación en Obstrucción Pulmonar. Cuestionario Platino México. [Consultado 26 de abril de $20 \mathrm{II}$. Disponible en www.platino-alat.org/docs/ cuestionario_platino_ mexico.pdf

16. The International Study of Asthma and Allergies in Childhood (ISAAC) Steering Committee. Worldwide variation in prevalence of symptoms of asthma, allergic rhinoconjunctivitis, and atopic eczema: ISAAC. Lancet I998;35I:I225-I232

17. Vargas MH, Sienra-Monge J], Díaz-Mejía G, DeLeón-González M. Asthma and geographical altitude: an inverse relationship in Mexico. J Asthma 1999;36:5 II-5I7.

18. Wilson DH, Adams RJ, Tucker G, Appleton S, Taylor AW, Ruffin RE. Trends in asthma prevalence and population changes in South Australia, 1990-2003. Med J Aust 2006;|84:226-229.

19. Guddattu V, Swathi A, Nair NS. Household and environment factors associated with asthma among Indian women: a multilevel approach. J Asthma 2010;47:407-4II.

20. Sood A, Cui X, Qualls C, Beckett WS, Gross MD, Steffes MW, et al. Association between asthma and serum adiponectin concentration in women. Thorax 2008; 63:877-882.

2I. McLachlan CR, Poulton R, Car G, Cowan J, Filsell S, Greene JM, et al. Adiposity, asthma, and airway inflammation. J Allergy Clin Immunol 2007; 1 19:634-639.

22. Sood A. Sex differences: implications for the obesity-asthma association. Exerc Sport Sci Rev 201 I;39:48-56.

23. Sood A, Qualls C, Li R, Schuyler M, Beckett WS, Smith LJ, et al. Lean mass predicts asthma better than fat mass among females. Eur Respir 20II;37:65-7I.

24. Global Initiative for Chronic Obstructive Lung Disease Global Strategy for the Diagnosis, Management, and Prevention of Chronic Obstructive Pulmoňary Disease (2006). [consultado el 26 de junio de 201 I] Disponible en: http://www.who.int/respiratory/copd/GOLD_WR_06.pdf 25. Kobylianskii VN, Babadzhanova G, Suntsov I. Investigation into relationship between chronic obstructive pulmonary disease, bronchial asthma, and type 2 diabetes. Klin Med (Mosk) 2009;87:40-43.

26. Fujiwara Y, Arakawa T. Epidemiology and clinical characteristics of GERD in the Japanese population. J Gastroenterol 2009;44:5 I8-534. 27. Song Y, Klevak A, Manson JE, Buring JE, Liu S. Asthma, chronic obstructive pulmonary disease, and type 2 diabetes in the Women's Health Study. Diabetes Res Clin Pract 2010;90:365-37I. 28. Wertz DA, Pollack M, Rodgers K, Bohn RL, Sacco P, Sullivan SD. Impact of asthma control on sleep, attendance at work, normal activities, and disease burden. Ann Allergy Asthma Immunol 2010;105:I I8-123. 